and received standardised rehabilitation. Blinded outcome assessments were at $4,7,13$, and 24 weeks. The primary outcome was muscle-tendon function assessed by work performed during the heel-rise endurance test (HRET), measured with the Limb Symmetry Index (LSI) $(0-100 \% ; 100 \%$ denotes full recovery) at 24 weeks. Secondary outcomes were the Achilles Tendon Rupture Score (ATRS), quality of life (SF-12), pain and goal attainment. The trial was prospectively registered.

Results Of 230 participants, 114 were allocated to PRP injection (103 received PRP), 116 were allocated to and received placebo. At 24 weeks, 201/230 (87\%) completed the HRET and 214/230 (93\%) completed patient reported outcomes. Participant characteristics between the groups were similar. There was no difference between groups at 24 weeks in LSI (mean difference $=-4.373 ; 95 \% \mathrm{CI}-11.217$ to $2.471 ; \mathrm{p}=0.195$ ). There were no differences in the secondary outcomes and adverse event rates.

Conclusion This trial design and standardised PRP preparation secure the first robust clinical trial evidence for PRP in managing Achilles tendon rupture, and suggest that PRP offers no patient benefit. The use of PRP in soft tissue injuries must be questionable unless supported by equally robust evidence indicating positive outcomes.

\section{RELATIONSHIP BETWEEN CHILDHOOD OVERWEIGHT/ OBESITY INDICATORS AND VIGOROUS PHYSICAL ACTIVITY AMONG NHANSE III BOYS (8 TO 11 YEARS OLD): DIFFERENT INTERPRETATION BETWEEN BODY MASS INDEX AND SUBCUTANEOUS ADIPOSITY SIZE AND SHAPE}

H Kim, E Petherick, I Varela-Silva. Loughborough University, UK

\subsection{6/bjsports-2019-BASEMAbs.4}

Aim The aim of this study was to investigate whether the relationship between BMI and vigorous physical activity (VPA) differs when both the overall and regional distribution of subcutaneous adiposity (SA) are considered as alternative fatness indicators of BMI.

Methods The sample data were obtained from the third National Health and Nutrition Examination Survey (NHANES III). Four skinfolds (triceps, subscapular, supraspinal, thigh), BMI, age, race/ethnicity, height, and VPA (i.e., times per week exercise made you sweat) data of 1,028 boys (8-11 yrs) were extracted. The overall and regional distribution of the four skinfolds were calculated into Subcutaneous Adiposity Size and Shape (SASS) variables based on the description provided in Healy and Tanner's method (1): transform all four skinfolds into logarithms, 2) calculate average (SA-size estimation), 3) calculate difference between the average and each log-transformed skinfold, 4) run principal component analysis (SAshape estimation). The samples were categorized into three groups based on participants' VPA levels (very-active: VPA $>5$ times, active: $2<\mathrm{VPA}<4$ times, non-active: $\mathrm{VPA}<1$ time). Then, MANCOVA/ANCOVA (BMI) were conducted separately to ascertain whether the fatness indicators provide consistent interpretations of the relationship with VPA.

Results The MANCOVA showed significant interaction between the SASS and VPA $(p<0.05)$. The MANCOVA Bonferroni-adjustment results indicated that SA-size $(\mathrm{F}(2,938)$ $=5.528, \mathrm{p}<0.005$; partial $\eta 2=0.012)$ and subscapular-to-thigh SA-shape was significantly associated with VPA $(\mathrm{F}(2,938)$ $=9.587, \mathrm{p}<0.0005$; partial $\eta 2=0.020)$, but not triceps- supraspinal SA-shape $(\mathrm{F}(2,938)=1.485, \mathrm{p}=0.227$; $\quad$ partial $\eta 2=0.003)$. This means that the very active and active boys had significantly larger SA-size and larger thigh and smaller subscapular SA-shape than the non-active boys. The ANCOVA result showed no BMI difference between the VPA groups ( $F$ $(2,1024)=1.296, p=0.274)$.

Conclusion It was concluded that the interpretations made via SASS and BMI when associated with VPA are different. Future studies need to further investigate whether tackling childhood obesity by focusing on BMI-based obesity is affective approach to reduce body fat.

\section{REFERENCE}

1. Healy MJR, Tanner JM. Size and shape in relation to growth and form. Symp Zool Soc Lond 1981;46:19-35.

\section{THE MOTIVATORS AND BARRIERS TO PHYSICAL ACTIVITY IN PRIMARY PREVENTION OF CHRONIC DISEASE}

A Pavitt. University of Oxford, UK

\subsection{6/bjsports-2019-BASEMAbs.5}

Background Regular physical activity is protective against many chronic diseases. Despite this, 39\% of adults in the UK are insufficiently active with an increased risk of chronic disease. Moving Medicine is an online resource developed by the FSEM to facilitate clinician-led conversations to reduce the risk of chronic disease through increased physical activity.

Aim This is the first review to categorise and understand the principle motivators for and barriers against physical activity, for the primary prevention of chronic disease. It has informed the development of a national Moving Medicine resource.

Methods A structured search of MEDLINE and OpenGrey, augmented by handsearching of reference lists and contents of key journals. Qualitative evidence was then synthesised thematically.

Results Thirteen papers were selected as being of good quality (structured interviews and focus groups rather than questionnaires) and relevant to the aims of this review. Motivators identified were grouped into: anticipated positive effects, support/encouragement, social aspects, environmental aspects, goal-setting, and the use of feedback. Barriers to physical activity were grouped into: time constraints, physical health problems, environmental aspects, cost and poor communication.

Building results into the resource The results of this review were presented to an expert panel of SEM doctors, GPs, nurses, physiotherapists, and patients. This multidisciplinary team then helped shape the primary prevention resource on the Moving Medicine website. See examples from the resource below.

Conclusion Good quality conversations on physical activity in healthcare require an understanding of the key motivators and barriers reported by patients. Combining this qualitative review with the knowledge, skills and the experience of a multidisciplinary expert panel has enabled the development of a unique patient-centred primary prevention resource. Delivering this information through MovingMedicine.ac.uk is a vital step in using these important findings to inform routine clinical practice across the NHS. This should facilitate an increase in the proportion of adults meeting recommended physical activity levels and therefore reduce the rates of chronic disease. 